\title{
Wilms tumor and Colorectal Adenocarcinoma an Unusual Concurrence
}

\author{
Dr. Pallavi Srivastava ${ }^{1}$, Prof Nuzhat Husain ${ }^{2 *}$, Dr. Saumya Shukla ${ }^{3}$, Dr. Vani Gupta ${ }^{4}$ \\ ${ }^{1}$ Senior Resident, Department of Pathology, Dr Ram Manohar Lohia Institute of Medical Sciences Lucknow, U.P, India \\ ${ }^{2}$ Prof and Head, Department of Pathology, Dr Ram Manohar Lohia Institute of Medical Sciences Lucknow, U.P, India \\ ${ }^{3}$ Assistant Professor, Department of Pathology, Dr Ram Manohar Lohia Institute of Medical Sciences Lucknow, U.P, India \\ ${ }^{4}$ Senior Resident, Department of Pathology, Dr Ram Manohar Lohia Institute of Medical Sciences Lucknow, U.P, India
}

\begin{abstract}
DOI: $10.36348 /$ sipm.2020.v05i04.003
| Received: 07.04.2020 | Accepted: 14.04.2020 | Published: 21.04 .2020

*Corresponding author: Prof Nuzhat Husain
\end{abstract}

\section{Abstract}

Multiple synchronous primary malignancies have been reported in colon carcinoma and studied for lynch syndrome or constitutional mismatch repair deficiency syndrome, however we present first case of co-occurance of colorectal adenocarcinoma and wilms tumor with mismatch repair deficiency. A 17 year old girl presented with pain in abdomen. A computerized tomography of whole abdomen revealed a large left sided renal mass along with mass lesion seen in the rectum Histopathology from both the mass revealed presence of colorectal adenocarcinoma and wilms tumor in renal mass. Immunohistochemistry for microsatellite instability was performed which showed loss of expression for MSH2/MSH6 and absence of BRAF mutation with presence of KRAS mutation. Diagnosis of wilms tumor with colorectal adenocarcinoma was made and possibility of constitutional mismatch repair deficiency was considered. A multidisciplinary approach is required for diagnosing synchronous malignancies and apart from clinical, radiological and histopathological studies, microsatellite instability testing in patients with colorectal carcinoma should also be considered to rule out Lynch syndrome or Constitutional Mismatch Repair Deficiency Syndrome. This report describes for the first time the coexistence of a colon carcinoma with Wilms tumor.

Keywords: Colorectal carcinoma, Lynch syndrome, Constitutional mismatch repair deficiency syndrome.

Copyright @ 2020: This is an open-access article distributed under the terms of the Creative Commons Attribution license which permits unrestricted use, distribution, and reproduction in any medium for non-commercial use (NonCommercial, or CC-BY-NC) provided the original author and sources are credited.

\section{INTRODUCTION}

Multiple primary tumor have shown an increasing trend in recent times. The primary tumor may be synchronous or metachronous. There are various definition and according to the Surveillance Epidemiology and End Results (SEER) database occurrence of two primary malignancies within a 2month period should be considered as synchronous while more than 2 month considered as metachronous multiple primaries [1] however the rules according to the IARC suggest an interval of less than 6 months for synchronous multiple primaries (or metachronous if more than 6 months) if arising in different sites [2]. The incidence of multiple primary malignances varies from $2-17 \%$, various risk factors have been studied however the risk factors incudes inherited predisposition to cancer; cancer-promoting aspects of lifestyle, hormonal and environmental factors; treatment of the previous primary cancer and increased surveillance of cancer survivors [2, 3]. Among the colorectal carcinoma (CRC), lynch syndrome constitutes around $3 \%$ of all the CRC. Lynch syndrome is an autosomal dominant condition and may be associated with various carcinoma at other sites, most commonly endometrial followed by urinary tract, ovary, small intestine, gastric, hepatobiliary tract, prostate, melanoma, ovarian germ cell, sarcoma and mesothelioma[4]. We present a case of CRC with synchronous presence of renal mass diagnosed as Wilm's tumor. CRC are among the most common second malignancy post treatment of Wilms tumor or Rhabdomyosaarcoma[5], however synchronous presence of both the malignancies have not been published in literature as yet. The tumor showed evidence of mismatch repair deficiency with loss of expression for MSH2 and MSH6 along with presence of KRAS mutation and negative expression for BRAFV600E mutation by IHC.

\section{CASE PRESENTATION}

A 17 years old girl presented to the Gastrosurgery department with chief complaints of pain in abdomen since 6 months and bleeding per rectum since 7 days. There was no history of hematuria, malena and hematochezia. No significant family history was evident. A computerized tomography whole abdomen revealed a large left sided renal mass occupying upper and mid pole measuring $184 \times 164 \times 142 \mathrm{~mm}$ in size. 
The lesion was compressing the spleen and greater curvature of stomach, pushing the body and tail of pancreas. The renal artery and vein is pushed inferiorly and drapped around the mass.(Figure 1A) Another mass lesion is seen in the rectum at a distance of $\sim 8.5 \mathrm{~cm}$ from anal verge measuring $\sim 26 \mathrm{x} 25 \mathrm{~mm}$ in size, perirectal fat stranding is also seen along with perirectal lymph nodes (Figure1B). Few expansile lytic lesions are seen in the iliac bone suggestive of metastasis. A small biopsy was taken from the rectal lesion and sent for histopathological examination which was reported as adenocarcinoma rectum. A renal scan was also performed which revealed adequate renal drainage and clearance. Radical nephrectomy and lower anterior resection was performed and sent for histopathological examination. Grossly a large renal mass was received, tumor was seen occupying the upper pole and mid pole, a rim of unremarkable renal parenchyma was evident at lower pole.(Figure 2A) The mass was largely necrotic, friable, tan with areas of haemorrhage. The resection margins including ureteric resection margin and renal vessels were free of tumor along with renal sinus fat, renal capsule and gerota's fascia. The lower anterior resection specimen was also received which showed and ulceroinfiltrative growth involving the upper and mid part of rectum measuring $3.2 \times 3.0 \mathrm{~cm}$ in size involving $360^{\circ}$ circumference (Figure $2 \mathrm{~B}$ ), extending to pericolorectal tissue, however the circumferential resection margin were free. Hematoxylin and eosin stained section from both the specimen were studied. The section from the renal mass showed tumor composed of undifferentiated blastema comprising of densely packed primitive small blue cells with round to oval hyperchromatic overlapping nuclei and scanty cytoplasm, epithelial elements comprising of abortive tubules lined by elongated to ovoid irregular nuclei with moderate amount of amphophilic cytoplasm and fibroblast-like stroma was also evident, diagnosis of Triphasic Wilms tumor was made on histomorphological findings.(Figure 3A-C) The section from rectal mass showed ulcerated rectal mucosa with tumor disposed in gland and acini. Tumor was seen infiltrating the pericolorectal adipose tissue.(Figure 3DF) 12 out of 19 regional lymph node were positive for metastasis. The tumor were staged according to AJCC $8^{\text {th }}$ edition and reported as pT3N2. Immunohistochemistry (IHC) was also performed for detection of lynch syndrome, tumor showed loss of expression for MSH2 and MSH6 with intact expression for MLH1 and PMS2 and reported to have high probability for lynch syndrome (Figure 3G, H). Further IHC was performed for detection of BRAF mutation, by IHC the tumor showed negative expression for BRAFv600E mutation.(Figure3I) KRAS mutation test by PCR was also performed and was positive. Due to the unusual co-occurance of these malignancies possibility of constitutional mismatch repair deficiency (CMMRD) was considered as loss of expression for MSH2/MSH6 was seen in neoplastic as well as surrounding normal cells which is unlikely in lynch syndrome. The post-operative period was uneventful, however no further molecular testing to confirm CMMRD could be performed as patient died 1 month after surgery.

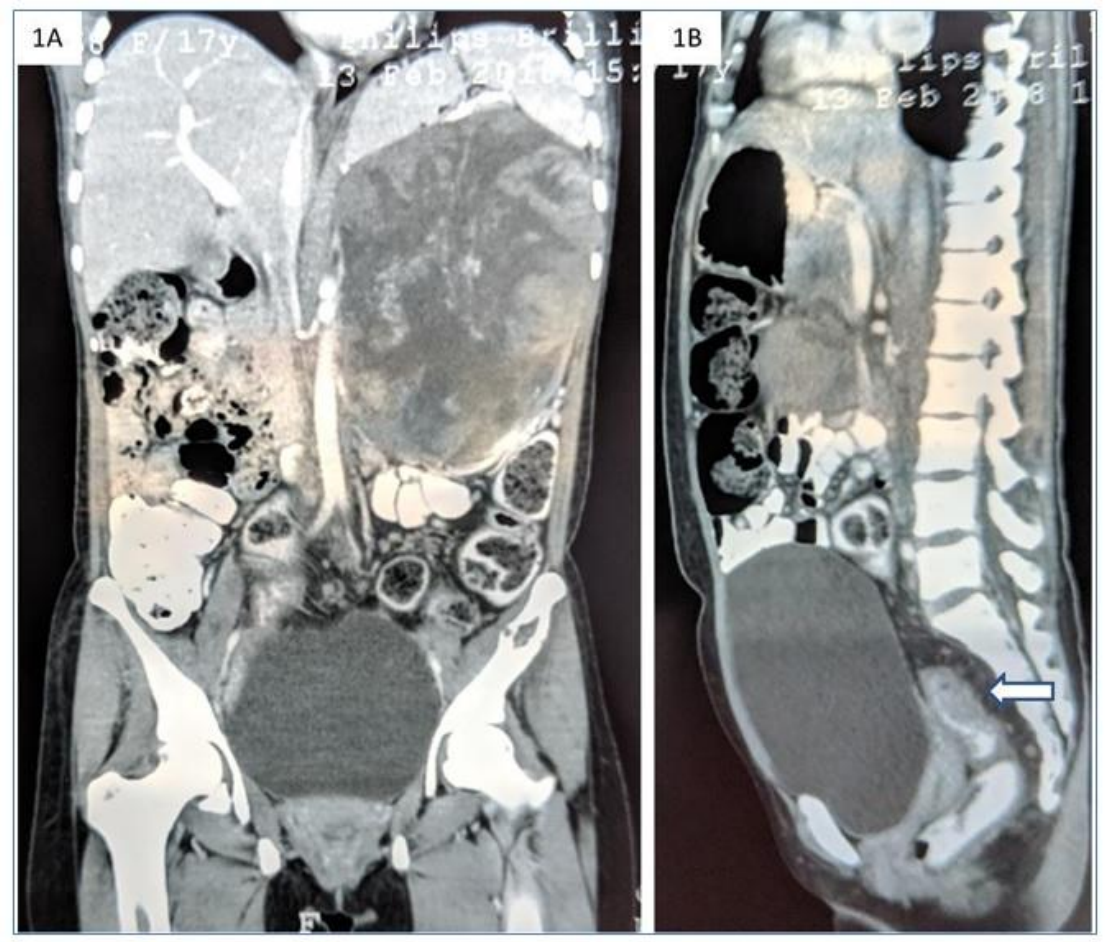

Fig-1: Imaging findings: A CECT whole abdomen showing large left sided renal mass [A], another mass lesion is seen in the rectum marked by arrow [B] 


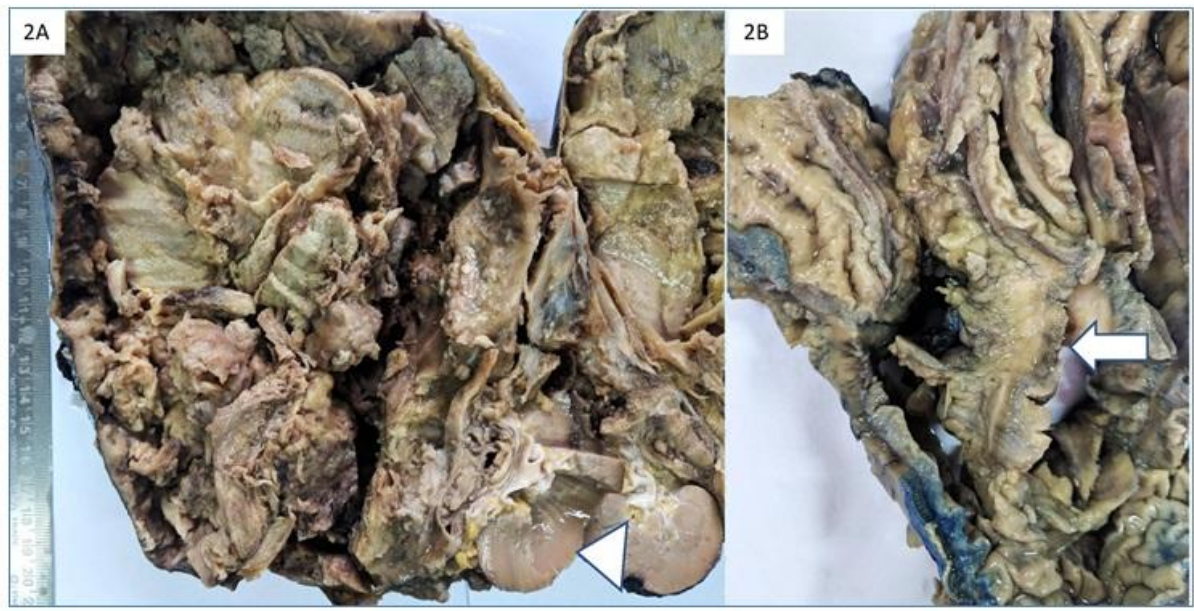

Fig-2: Gross findings: Image showing large renal mass occupying the upper pole and mid pole, a rim of unremarkable renal parenchyma was evident at lower pole marked by arrow head [A] The lower anterior resection specimen showing ulceroinfiltrative growth involving the upper and mid part of rectum $[B]$

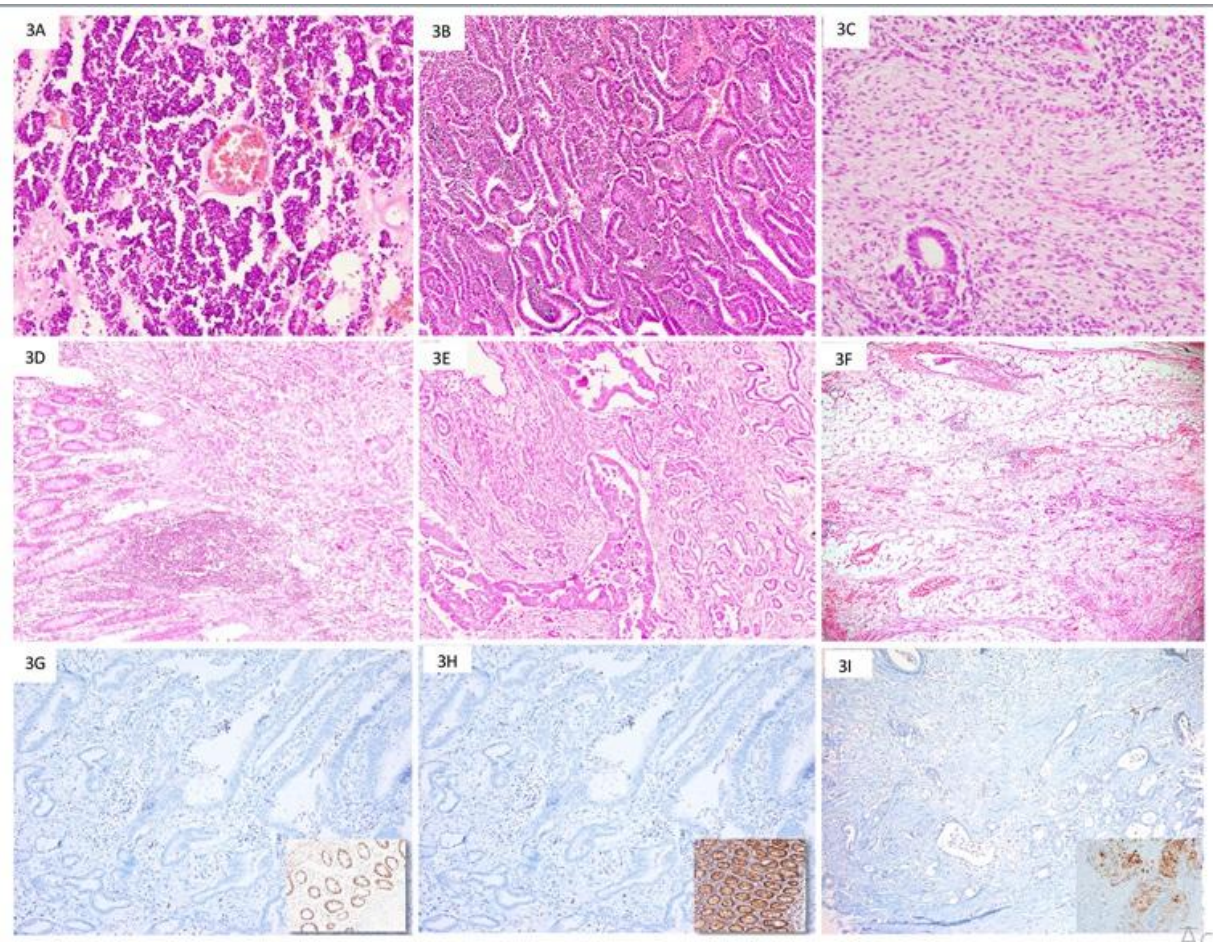

Fig-3:Histopathologic findings: The section from the renal mass showed tumor composed of undifferentiated blastema comprising of densely packed primitive small blue cells[A] epithelial elements comprising of abortive tubules $[B]$ and fibroblast-like stroma also evident $[C]$, the section from rectal mass showed ulcerated rectal mucosa with tumor disposed in gland and acini infiltrating the pericolorectal adipose tissue,

[D-F] Immunohistochemistry findings showing loss of expression for MSH2/MSH6 in neoplastic as well as surrounding normal cells with control tissue shown in inset[G,H] \& loss of BRAF expression[I]

\section{DISCUSSION}

Synchronous malignancies have been well documented in literature; the current case is an addition to list of synchronous malignancies. There is no well documented etiology and pathogenesis for the occurrence of multiple synchronous primaries, the bi and multi directional association among malignancies might be driven by a genetic predisposition (mismatch repair defect), common environmental risk factors (smoking, diet, carcinogens) or by the effect of treatment of one type of cancer on the other. In the current case we could detect mismatch repair deficiency by loss of expression for MSH2 and MSH6 in rectal mass and it is well established that various mismatch repair genes are functionally affected disposing individual to an increased risk of developing extracolonic tumors, including endometrial, ovarian, ureteral, and renal cancers including clear cell, papillary and chromophobe variant [6, 7]. In view of bi directional association, wilms tumor also present with synchronous malignancies with reported incidences of bilateral wilms tumor which constitute $4 \%$ to $7 \%$ of all Wilms tumours (WTs) with $22 \%$ associated with genitourinary abnormalities, aniridia, WAGR (WT, aniridia, genitourinary anomalies, and retardation) syndrome, Denys-Drash syndrome, hemihypertrophy 
or one of the other overgrowth syndromes[8] along with synchronous occurrence of other malignancies including Hepatocellular carcinoma, fibrolamellar variant, ganglioneuroma and neuroblastoma [9-11]. Densmore et al. [5] have also documented the phenomenon of early onset of adult type tumors associated with childhood solid tumors treated by radiotherapy and found that childhood tumors including Wilms tumor and Rhabdomyosarcoma presented with CRC post radiotherapy, however they presented after an interval of more than 10 years unlike the current case which had no pre-treatment history.

Biallelic mutations of MMR genes cause a distinct autosomal recessive inherited cancer predisposition namely CMMRD syndrome. CMMRD syndrome have documented to be associated with wilms tumor along with other malignancy including leukemia, lymphoma, brain tumors and rhabdomyosarcoma, 146 cases of CMMRD from 91 families have been documented however synchronous presence of wilms tumor with colorectal carcinoma has not been documented yet[12]. An interesting finding which further supported the diagnosis of CMMRD syndrome in the present case was loss of expression for MSH2/MSH6 in neoplastic as well as non-neoplastic surrounding stromal cells, hence such cases should not be considered as failure of proper staining and a staining control from different individual should be taken, as was done in the present case [12]. Negative BRAF expression also further supported the germline mutation as BRAF mutation favours sporadic pathogenesis [13]. The practicing physicians and pathologist should have a high grade of suspicion of the risk of synchronous malignancies in CRC and approach patients with diligence. Genetic counselling should be offered to patient as well as the family members for mutation analysis for lynch syndrome and CMMRD syndrome.

\section{CONCLUSION}

A multidisciplinary approach is required for diagnosing synchronous malignancies and apart from clinical, radiological and histopathological studies, microsatellite instability testing in patients with CRC should also be considered which is a cost-effective, feasible and less time consuming method. Possibility of CMMRD show also be considered along with lynch syndrome in CRC patients. This report describes the coexistence of a colon carcinoma with Wilms tumor and such cases highlights the need to perform routine pre-operative imaging studies to exclude synchronous asymptomatic tumors in patients with colorectal cancer, and detecting genetic aberrations after surgery with help of immunohistochemistry and molecular studies. The treatment strategy of synchronous and metachronous malignancies needs further research and with the advent of widespread gain of knowledge on patients with hereditary cancer and cancer survivors will hopefully help in the development of specific management and surveillance measures.

\section{REFERENCES}

1. Amer MH Multiple neoplasms, single primaries, and patient survival. Cancer Manag Res. 2014;6:119-34.

2. Coyte A, Morrison DS, McLoone P. Second primary cancer risk - the impact of applying different definitions of multiple primaries: results from a retrospective population-based cancer registry study. BMC Cancer. 2014;14

3. Soerjomataram I, Coebergh JW. Epidemiology of multiple primary cancers. Methods Mol Biol. 2009;471:85-105.

4. Lynch HT, de la Chapelle A. Hereditary colorectal cancer. N Engl J Med. 2003;348:919

5. Densmore T, Langer J, Molleston J, Dehner L, Coffin C. Colorectal adenocarcinoma as a second malignant neoplasm following Wilms tumor and rhabdomyosarcoma. Medical and Pediatric Oncology. 1996;27(6):556-560.

6. Mydlo JH, Agins JA, Donohoe J, Grob BM. A review of urologic cancer patients with multiple primary malignancies. World J Urol. 2001;19:240 243.

7. Papalampros A, Petrou A, Mantonakis E. Coexistence of a colon carcinoma with two distinct renal cell carcinomas: a case report. J Med Case Rep. 2011;5(1).

8. Indolfi P, Jenkner A, Terenziani M, Crocoli A, Serra A. Synchronous bilateral Wilms tumor: a report from the Associazione Italiana Ematologia Oncologia Pediatrica (AIEOP). Cancer. 2013;119(8):1586-92.

9. Maitra A, Ramnani D, Margraf L, Gazdar A. Synchronous Wilms Tumor and Fibrolamellar Hepatocellular Carcinoma: Report of a Case. Pediatric and Developmental Pathology. 2000;3(5):492-496.

10. Sarin, Y. K., Thakkar, N. C., Sinha S. Synchronous Ipsilateral Wilms Tumor and Neuroblastoma in an Infant. APSP J Case Rep 2016; 7(1):2

11. Geoerger B, Hero B, Harms D. Metabolic activity and clinical features of primary ganglioneuromas. Cancer 2001;91:1905-1913

12. Wimmer K, Kratz CP, Vasen HFA. Diagnostic criteria for constitutional mismatch repair deficiency syndrome: suggestions of the European consortium 'Care for CMMRD' (C4CMMRD). Journal of Medical Genetics. 2014; 51:355-365.

13. Sepulveda A. Molecular Biomarkers for the Evaluation of Colorectal Cancer Guideline From the American Society for Clinical Pathology, College of American Pathologists, Association for Molecular Pathology, and American Society of Clinical Oncology. Arch Pathol Lab Med. 2017; 141:625-657. 